\title{
Wigner flow reveals topological order in quantum phase space dynamics
}

\author{
Ole Steuernagel, Dimitris Kakofengitis and Georg Ritter \\ School of Physics, Astronomy and Mathematics, University of Hertfordshire, Hatfield, AL10 9AB, UK ${ }^{*}$
}

(Dated: December 5, 2012)

\begin{abstract}
The behaviour of classical mechanical systems is characterised by their phase portraits, the collections of their trajectories. Heisenberg's uncertainty principle precludes the existence of sharply defined trajectories, which is why traditionally only the time evolution of wave functions is studied in quantum dynamics. These studies are quite insensitive to the underlying structure of quantum phase space dynamics. We identify the flow that is the quantum analog of classical particle flow along phase portrait lines. It reveals hidden features of quantum dynamics and extra complexity. Being constrained by conserved flow winding numbers, it also reveals fundamental topological order in quantum dynamics that has so far gone unnoticed.
\end{abstract}

PACS numbers: 03.65.-w, 03.65.Ta

Phase portraits characterise the time evolution of dynamical systems and are widely used in classical mechanics 11. For the conservative motion of a single particle, moving in one dimension $x$ under the influence of a static smooth potential $V(x)$ only, the classical Liouville flow in phase space is regular 2 and largely determined by location and nature of its flow stagnation points. Situated on the $x$-axis wherever the potential is force-free (momentum $p=0$ and $-\partial V / \partial x=0$ ), the local flow forms clockwise vortices around stagnation points at minima of the potential, maxima split the flow and therefore lie at the intersections of flow separatrices, saddle points of the potential lead to an elongated saddle flow pattern oriented along the $x$-axis.

Here we investigate the quantum dynamics of bound states of non-harmonic potentials; their quantum phase space flow reveals rich non-classical features:

Dependence of flow on the state of the system [3] leading to directional deviation from classical trajectories [4] and flow reversal [5], time-dependent quantum-displacement of classical stagnation points [6], occurrence of additional non-classical stagnation points (see Fig. 1 below) whose positions change over time [6] (even for conservative systems), and conservation of the flow orientation winding number $\omega$, see eq. (6) below, carried by all flow stagnation points during all stages of their time evolution including instances when they split from or merge with other stagnation points.

For a single quantum particle described by a complex time-dependent amplitude function $\psi(x ; t)$ the associated quantum analog of classical phase space probability distributions is Wigner's function $W(x, p ; t)$ 7, 8, with $p$ the particle's momentum. Structurally, $W$ is a Fouriertransform of the off-diagonal coherences of the quantum system's density matrix $\varrho$, i.e.

$$
W(x, p ; t)=\frac{1}{\pi \hbar} \int_{-\infty}^{\infty} d y \varrho(x+y, x-y ; t) \cdot e^{\frac{2 i}{\hbar} p y},
$$

where $\hbar=h /(2 \pi)$ is Planck's constant. Unlike $\psi$ or $\varrho$, the Wigner function only assumes real values, but these do become negative [7, 9], defying description in terms of classical probability theory [9-11, thus revealing quantum aspects of a system [12.

The time evolution of $W$ can be cast in the form of a flow field $\boldsymbol{J}(x, p ; t)$ [13, the 'Wigner flow' 4, which describes the flow of Wigner's quasi-probability density in phase space. It has the two components

$$
\boldsymbol{J}=\left(\begin{array}{c}
J_{x} \\
J_{p}
\end{array}\right)=\left(\begin{array}{c}
\frac{p}{m} W(x, p ; t) \\
-\sum_{l=0}^{\infty} \frac{(i \hbar / 2)^{2 l}}{(2 l+1) !} \frac{\partial^{2 l} W(x, p ; t)}{\partial p^{2 l}} \frac{\partial^{2 l+1} V(x)}{\partial x^{2 l+1}}
\end{array}\right),
$$

fulfilling Schrödinger's equation which takes the form

$$
\frac{\partial W}{\partial t}+\frac{\partial J_{x}}{\partial x}+\frac{\partial J_{p}}{\partial p}=0
$$

of a continuity equation [7. Thus Wigner flow is the equivalent of classical Liouville flow, it has, so far, not been studied in great detail [4, 13, 15].

Non-locality [3, 16] originates both in definition (1) of the Wigner function and the higher derivatives of $V$ occurring in the Wigner flow (2).

The marginals of the Wigner function yield the probability distributions in position $|\psi(x ; t)|^{2}$ and momentum $|\phi(p ; t)|^{2}$, see Fig. 2 and references [7, 9, 17]. Integrating over the expressions in the continuity equation analogously shows that the marginals of the Wigner flow yield the respective probability currents in $x$ and $p$

$$
\begin{aligned}
\frac{d}{d t}\left|\psi(x ; t)^{2}\right| & =\int_{-\infty}^{\infty} d p J_{x}(x, p ; t)=\hat{\jmath}_{x}(x ; t) \\
\text { and } \quad \frac{d}{d t}\left|\phi(p ; t)^{2}\right| & =\int_{-\infty}^{\infty} d x J_{p}(x, p ; t)=\hat{\jmath}_{p}(p ; t),
\end{aligned}
$$

where $\phi(p)$ is the momentum representation of $\psi(x)$. While the $x$-component can be rewritten as the probability current $\hat{\jmath}_{x}(x ; t)=\frac{\hbar}{2 i m}\left(\psi^{*} \partial_{x} \psi-\psi \partial_{x} \psi^{*}\right)$, in general, no such simple expression exists for $\hat{\jmath}_{p}(p ; t)$.

The dynamics of the harmonic potential, the most studied quantum case, e.g. of quantum optics [9, 11], amounts to a rigid rotation of the Wigner function

* O.Steuernagel@herts.ac.uk 


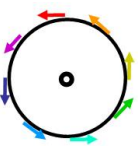

$\omega=+1$

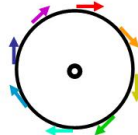

$\omega=+1$

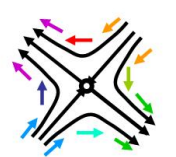

$\omega=-1$

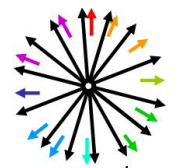

$\omega=+1$

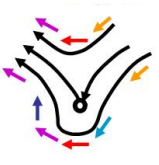

$\omega=0$
FIG. 1. (color online) Flow field around various types of stagnation points of Wigner flow with associated winding numbers. This list is non-exhaustive.

around the origin of phase space. Only at the origin can a flow vortex form (Fig. 3 in Ref. [4]), just like in the classical case. The quantum harmonic oscillator and its isomorphism [18, the free quantum particle [19], constitute exceptional, degenerate cases where lines of stagnation of Wigner flow occur, and not only isolated stagnation points. This is due to the fact that for $V \propto x^{2}$ or $V=$ const. we have in eq. 22 $J_{p}=-W \frac{\partial V}{\partial x}$, just as in the 'classical limit' $(\hbar \rightarrow 0)$ and consequently with $W=0$ we always find $|\boldsymbol{J}|=0$. Because of these three facts (rigid rotation, classical form of $J_{p}$ and line formation) the non-classical phase space features discussed here cannot be seen in the degenerate cases primarily studied so far [9, 11, 19].

The degeneracies of the degenerate cases leading to the formation of stagnation lines are lifted for non-harmonic potentials by the presence of terms with $l>0$ in $J_{p}$
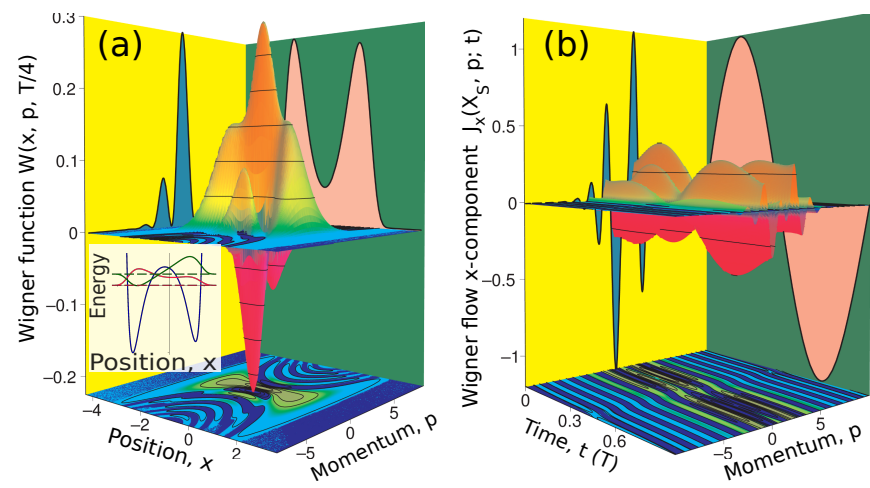

FIG. 2. (color online) Wigner function, Wigner flow and momentum distribution of state $\Psi$ (parameters $\hbar=1, m=$ $1 / 2, \alpha=0.5, \Delta E=0.5)$. (a) Wigner function $W(x, p ; T / 4)$, projection to bottom shows its contours. Projections onto background walls show momentum and position probability distribution (blue and rose filled curves, respectively, in arbitrary units). Inset: Plot of Caticha-potential with wave functions for lowest two energy eigenstates shifted to their respective energy levels (dashed lines). (b) $x$-component of Wigner flow $J_{x}\left(X_{S}, p ; t\right)$ at barrier top at position $X_{S}$; bottom projection shows its contours (note its phase shift at $t=T / 2$ ). Projections onto background walls show time and momentum projections (blue and rose filled curves, $\left\langle J_{x}\left(X_{S}, p\right)\right\rangle_{T}=$ $\int_{0}^{T} d \tau J_{x}\left(X_{S}, p ; \tau\right)$ and $\hat{\jmath}_{x}\left(X_{S} ; t\right)$ of eq. 4, respectively, in arbitrary units). The projection $\hat{\jmath}_{x}$ features the sinusoidal variation $\propto \sin (2 \pi t / T)$ expected of the tunnelling current of a 2-state system. and leads to the formation of separate stagnation points instead. The boundedness and continuity of wave functions of infinitely differentiable non-harmonic potentials and the unitarity of such systems' quantum dynamics induces homotopies that keep all smooth changes of $\boldsymbol{J}$ in space and time around stagnation points smooth. We therefore conjecture that the topological structure of the Wigner flow field around stagnation points remains conserved. To monitor this we introduce the Wigner flow orientation winding number determined by the integral

$$
\omega(\mathcal{L} ; t)=\frac{1}{2 \pi} \oint_{\mathcal{L}} d \varphi
$$

along a closed (convex) loop $\mathcal{L}$; here $\varphi$ is the orientation angle between the positive $x$-axis and the Wigner flow 'vectors' $\boldsymbol{J}$. For 'empty' paths, not including a stagnation point of $\boldsymbol{J}, \omega=0$; for vortices $\omega=+1$, see Fig 2 The winding number $\omega(\mathcal{L} ; t)$ is unchanged under continuous path deformations that do not push $\mathcal{L}$ across a stagnation point and as long as time evolution does not move a stagnation point across the loop. It assumes integer values only (assuming the integration path $\mathcal{L}$ does not run through a stagnation point) and is conserved. The sum of winding numbers of all stagnation points within a loop is conserved, even when they split or coalesce: the stagnation points carry topological charge [20.

To give an example, we concentrate on Caticha's [21] smooth, slightly asymmetric, double well potential

$$
\begin{aligned}
V(x) & =1+E_{0}+\frac{3}{2} \Delta E-\Delta E \alpha \sinh (2 x) \\
& +\cosh ^{2}(x)\left(\frac{\Delta E^{2}}{4} \alpha \sinh (2 x)-\frac{\Delta E^{2}}{4}-2 \Delta E\right) \\
& +\frac{\Delta E^{2}}{4}\left(\alpha^{2}+1\right) \cosh ^{4}(x),
\end{aligned}
$$

featuring high outer walls, and wells separated by a barrier of sufficient height, such that at least ground and first excited energy eigenstate tunnel through it, Fig. 22(a) inset. To display all non-classical flow features listed in the introduction it suffices to investigate the balanced superposition

$$
\Psi(x ; t)=\frac{\psi_{0}(x) e^{-i E_{0} t / \hbar}-\psi_{1}(x) e^{-i\left(E_{0}+\Delta E\right) t / \hbar}}{\sqrt{2}},
$$

of ground $\psi_{0}(x)=\psi_{0} \cosh (x) \exp \left[-\frac{\Delta E}{4}\left(\cosh ^{2}(x)+\alpha x\right.\right.$ $\left.\left.+\frac{\alpha}{2} \sinh (2 x)\right)\right]$ and first excited state $\psi_{1}(x)=$ $\psi_{1}[\alpha+\tanh (x)] \psi_{0}(x)$, with energies $E_{0}$ and $E_{0}+\Delta E$ and normalisation constants $\psi_{0}$ and $\psi_{1}$, respectively [21]. Since the eigenstates are real functions their Wigner functions obey $W(x, p)=W(x,-p)$ which implies that

$$
\begin{aligned}
J_{x}(x, p) & =-J_{x}(x,-p) \\
\text { and } J_{p}(x, p) & =J_{p}(x,-p) .
\end{aligned}
$$

Their individual Wigner flow patterns, which the superposition $\Psi$ 'inherits', are displayed in the supplement [22]. All these Wigner functions have to be determined numerically [23]. Of the low energy states $\Psi$ is the 'most 


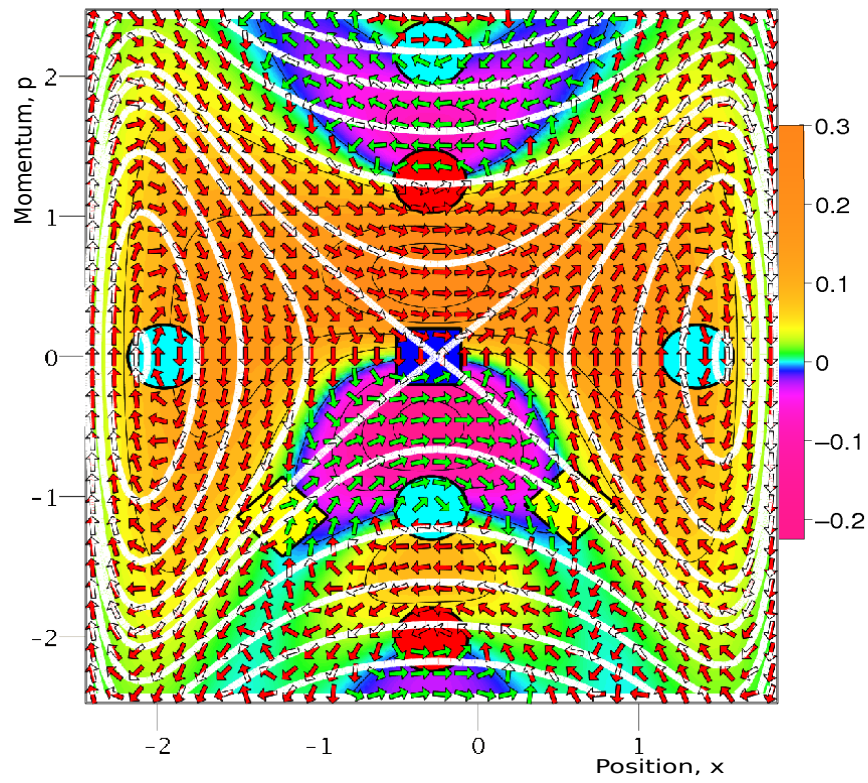

FIG. 3. (color online) Features of Wigner flow of an asymmetric double well potential (same parameters as in Fig. 1, at $t=T / 4)$. Contour plot of Wigner function $W(x, p ; T / 4)$ (black contour lines) with an overlay of coloured arrows showing normalised Wigner flow $\boldsymbol{J} /|\boldsymbol{J}|$ (red arrows for $W>0$ and green for $W<0$ ). The classical phase portrait is shown as a collection of thick white lines. All locations of Wigner flow stagnation points are highlighted by symbols (cyan and red circles centre on clockwise and anti-clockwise vortices, respectively, yellow diamonds on separatrix intersections, and the blue square on a $p$-directed saddle flow. The quantumdisplacement of the vortices near the potential minima, towards the center, is clearly visible.

dynamic' in that it shifts all of the particle's probability distribution back and forth between left and right well. Its Wigner function $W(t=T / 4)$ at a quarter of the tunnelling period time $(T=2 \pi \hbar / \Delta E)$ is displayed in Figs. 2(a) and 3

The Wigner flow's state dependence has two aspects: the negativity of $W$ reverses the flow, and the dependence of $J_{p}$ on $W$ and $V$ leads to sideways deviation of Wigner flow from classical phase portrait lines. In the case of eigenstates of the harmonic oscillator, the former leads to shear flow between neighbouring sectors of alternating polarity [4]. The latter deviation can remain mild for eigenstates of a weakly anharmonic potential 4], in our case it is very pronounced leading to the formation of non-classical vortices which are quantum-displaced off the $x$-axis and some of which spin anti-clockwise, Figs. 1 and 3. Indications of non-classical vortices seem to have been observed in 'chaotic' quantum systems before [24].

Flow reversal affects quantum tunnelling. The wave function of a particle, tunnelling through a barrier, is coherent across it, implying that interference fringes of the Wigner function form in the tunnelling region; orientated parallel to the $x$-axis [10, Figs. 2(b) and 3. Neighbouring phase space regions contain strips of alternat-

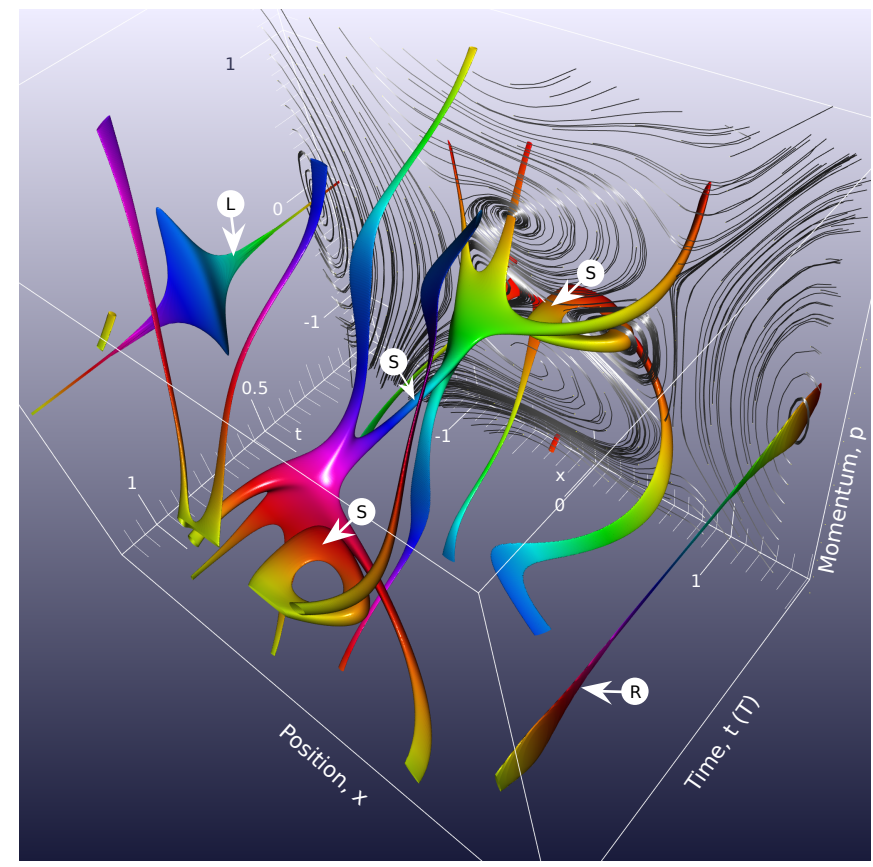

FIG. 4. (color online) Wigner flow's stagnation points' positions across phase space as a function of time. Same parameters as in Fig. 2. The tube surfaces show where in phase space the magnitude of Wigner flow is small $\boldsymbol{J}(x, p ; t)^{2}=3 \cdot 10^{-5}$. They are displayed over $120 \%$ of one oscillation period $(t=$ $-0.1 \cdot T \ldots 1.1 \cdot T)$; the rainbow spectrum is matched to $T$, redorange for $t=0$, via green, cyan at $t=0.5 T$, through blue and purple back to red-orange. Because of the periodicity of the two-state scenario, the red-orange-yellow torus is seen twice at beginning and end of the time window. At the core of all tubes lie time-lines of stagnation points. Their movement through phase space leads to their mergers and splits. The grey flow integration lines are guiding the eye past vortices and separatrices, they do not represent physical flow since they integrate $\boldsymbol{J}(x, p ; t=0.05)$ at fixed time. The $x$-coordinate is shown from left well minimum at $X_{L}=-2.095$ to right well minimum at $X_{R}=1.514$, the position of the associated vortices ((D) in left well and $(\mathbb{B}$ in right), at $p=0$ and just inside the plot region, confirms the inward quantum-displacement of these stagnation points. The remnant of the classical separatrix stagnation point at position $X_{S}+\delta x_{S}(t) \approx-0.3$ is labelled (S). It follows a bent path and becomes displaced, forming part of the torus, when it coalesces with or splits from other stagnation points. All mergers or splits are constrained by topological charge [20] conservation, see Fig. 5.

ing Wigner function polarity alternating their flow direction [6], Fig. 2(b). The further apart the two wells, the finer the interference pattern [10] and the stronger the resulting flow cancellation. Thus we find that tunnelling can be described as a transport phenomenon that plays out over large parts of quantum phase space [6, 25, frustrated by phase space interference.

For a low energy state, such as $\Psi$, the positions of remnant vortices originating from the potential's minima positions are quantum-displaced inward, towards the potential barrier, Figs. 3 and 4 . 

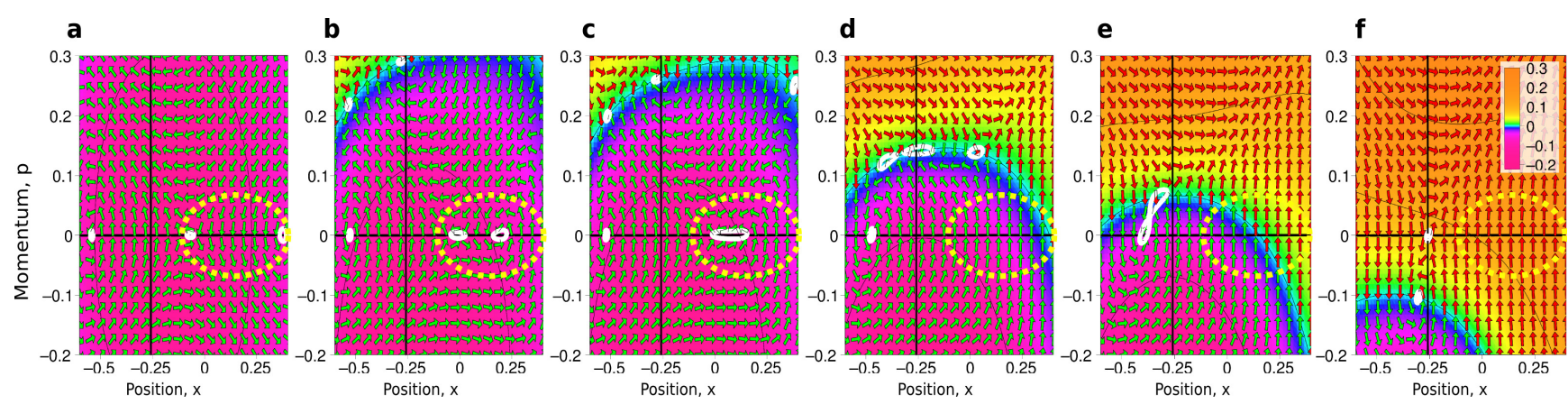

FIG. 5. (color online) Same system parameters as for Fig. 2. Contour plots of Wigner function with superimposed normalised flow field $\boldsymbol{J} /|\boldsymbol{J}|$ at times $t=0,0.075 T, 0.0875 T, 0.15 T, 0.2 T, 0.325 T$ in panels a to $\mathbf{f}$ demonstrates mergers and splitting of stagnation points and their pinning to zeros of the Wigner function. Each vertical black line shows the position $X_{S}$ of the tunnelling barrier top. Small white loops delineate areas where $\boldsymbol{J}^{2}=2 \cdot 10^{-6}$ and encircle stagnation points. We chose the region where the torus closes and several stagnation points coalesce (compare Fig. 4). The cross sections of the torus are visible in the right half of panels $\mathbf{a}, \mathbf{b}$ and $\mathbf{c}$, a convex loop $\mathcal{L}$ encircling both torus points only, such as the dotted yellow ellipse, yields $\omega=0$. This value is conserved over time throughout the merger which leads to the disappearance of the torus (panel $\mathbf{d}$ ). The mergers of the four stagnation points outside the yellow loop (two vortices and two separatrix crossings) in panels $\mathbf{d}$, e and $\mathbf{f}$ into one vortex and one separatrix crossing evidently carry a conserved total topological charge of zero throughout.

In classical physics stagnation points of phase space flow can only occur on the $x$-axis, here, according to eq. (2), they are pinned to the zero lines of the Wigner function, but occur off the $x$-axis, travel long distances and merge with or split from other stagnation points, see Figs. 4 and 5 .

For the Caticha potential, we observe a string of vortices with alternating handedness aligned in the $p$ direction located near the top of the tunnelling barrier, at position $X_{S}=-0.258$, Fig. 3. Over time they travel in the negative $p$-direction, Figs. 4 and 5 . When reaching the $x$-axis they coalesce with the remnant separatrix intersection point while the overall topological charge of the flow is conserved, see Fig. 5 .

To conclude: Wigner flow reveals non-classical features and added complexity of quantum phase space dynamics; at the same time it provides, through the conservation of the flow winding number $\omega$, the basis for an analysis of its topological invariants, ordering this complexity.
Systems that have been studied using quantum phase space techniques can be analysed using Wigner flow. Such systems arise [17] for example in chemical quantum dynamics [13, 14, 26], 'non-linear' quantum processes in closed single particle [15] or open multi-particle 27 29] systems, classical electromagnetic fields [30] and multiband semiconductor physics 31 .

\section{ACKNOWLEDGMENTS}

Use of the high-performance computing facility at the University of Hertfordshire's Science and Technology Research Institute is gratefully acknowledged. We thank Charles Young, Elias Brinks, Daniel Polani, Andreas Kukol and Cosmas Zachos for comments on the manuscript.
[1] D. D. Nolte, Phys. Today 63, 33 (2010).

[2] M. V. Berry, in Am. Inst. Phys. Conf. Ser. Vol. 46 (1978) pp. $16-120$.

[3] A. Donoso, Y. Zheng, and C. C. Martens, J. Chem. Phys. 119, 5010 (2003)

[4] H. Bauke and N. R. Itzhak, (2011), arXiv:1101.2683v1

[5] In degenerate quantum cases flow reversal can occur but none of the other non-classical flow patterns listed here.

[6] R. T. Skodje, H. W. Rohrs, and J. Vanbuskirk, Phys. Rev. A 40, 2894 (1989).

[7] E. Wigner, Phys. Rev. 40, 749 (1932).

[8] M. Hillery, R. F. O'Connell, M. O. Scully, and E. P. Wigner, Phys. Rep. 106, 121 (1984).

[9] W. P. Schleich, Quantum Optics in Phase Space (Wiley$\mathrm{VCH}, 2001)$.
[10] W. H. Zurek, Nature 412, 712 (2001), arXiv:quantph/0201118.

[11] P. Grangier, Science 332, 313 (2011)

[12] A. Ferraro and M. G. A. Paris, Phys. Rev. Lett. 108, $260403(2012)$

[13] A. Donoso and C. C. Martens, Phys. Rev. Lett. 87, $223202(2001)$

[14] K. H. Hughes, S. M. Parry, G. Parlant, and I. Burghardt, J. Phys. Chem. A 111, 10269 (2007)

[15] O. Gat, J. Phys. A: Math. Theo. 40, F911 (2007).

[16] J. S. Bell, Speakable and unspeakable in quantum mechanics. Collected papers on quantum philosophy (Cambridge University Press, 1987).

[17] C. K. Zachos, D. B. Fairlie, and T. L. Curtright, World Scientific 34 (2005), 10.1142/5287. 
[18] O. Steuernagel, (2010), arXiv:1008.3929.

[19] C. Kurtsiefer, T. Pfau, and J. Mlynek, Nature 386, 150 (1997)

[20] M. R. Dennis, K. O'Holleran, and M. J. Padgett, in Progress in Optics, Vol. 53, edited by E. Wolf (Elsevier, 2009) pp. $293-363$.

[21] A. Caticha, Phys. Rev. A 51, 4264 (1995)

[22] See Supplemental Material at [URL will be inserted by publisher] for the Wigner flow patterns of states $\psi_{0}$ and $\psi_{1}$.

[23] To determine the Wigner functions we integrated $y$ from $-2.7, \ldots, 2.7$. The summation cutoff for the determination of $J_{p}$ was chosen at $l_{\max }=12$.

[24] M. V. Berry and N. L. Balazs, J. Phys. A: Math. Gen. 12, 625 (1979).

[25] N. L. Balazs and A. Voros, Ann. Phys. 199, 123 (1990)

[26] L. L. Gibson, G. C. Schatz, M. A. Ratner, and M. J. Davis, J. Chem. Phys. 86, 3263 (1987)

[27] B. M. Garraway and P. L. Knight, Phys. Rev. A 50, 2548 (1994)

[28] I. Katz, R. Lifshitz, A. Retzker, and R. Straub, N. J. Phys. 10, 125023 (2008).

[29] S. Rips, M. Kiffner, I. Wilson-Rae, and M. J. Hartmann, N. J. Phys. 14, $023042(2012)$

[30] M. Levanda and V. Fleurov, Annals of Physics 292, 199 (2001), arXiv:cond-mat/0105137.

[31] O. Morandi, Phys. Rev. B 80, 024301 (2009) 


\title{
WIGNER FLOW REVEALS TOPOLOGICAL ORDER IN QUANTUM PHASE SPACE DYNAMICS
}

\author{
BY OLE STEUERNAGEL, DIMITRIS KAKOFENGITIS \& GEORG RITTER
}

In this supplement we display the Wigner flow patterns of the ground state $\psi_{0}$ and first exited state $\psi_{1}$ constituting the superposition state $\Psi$ of equation (8).

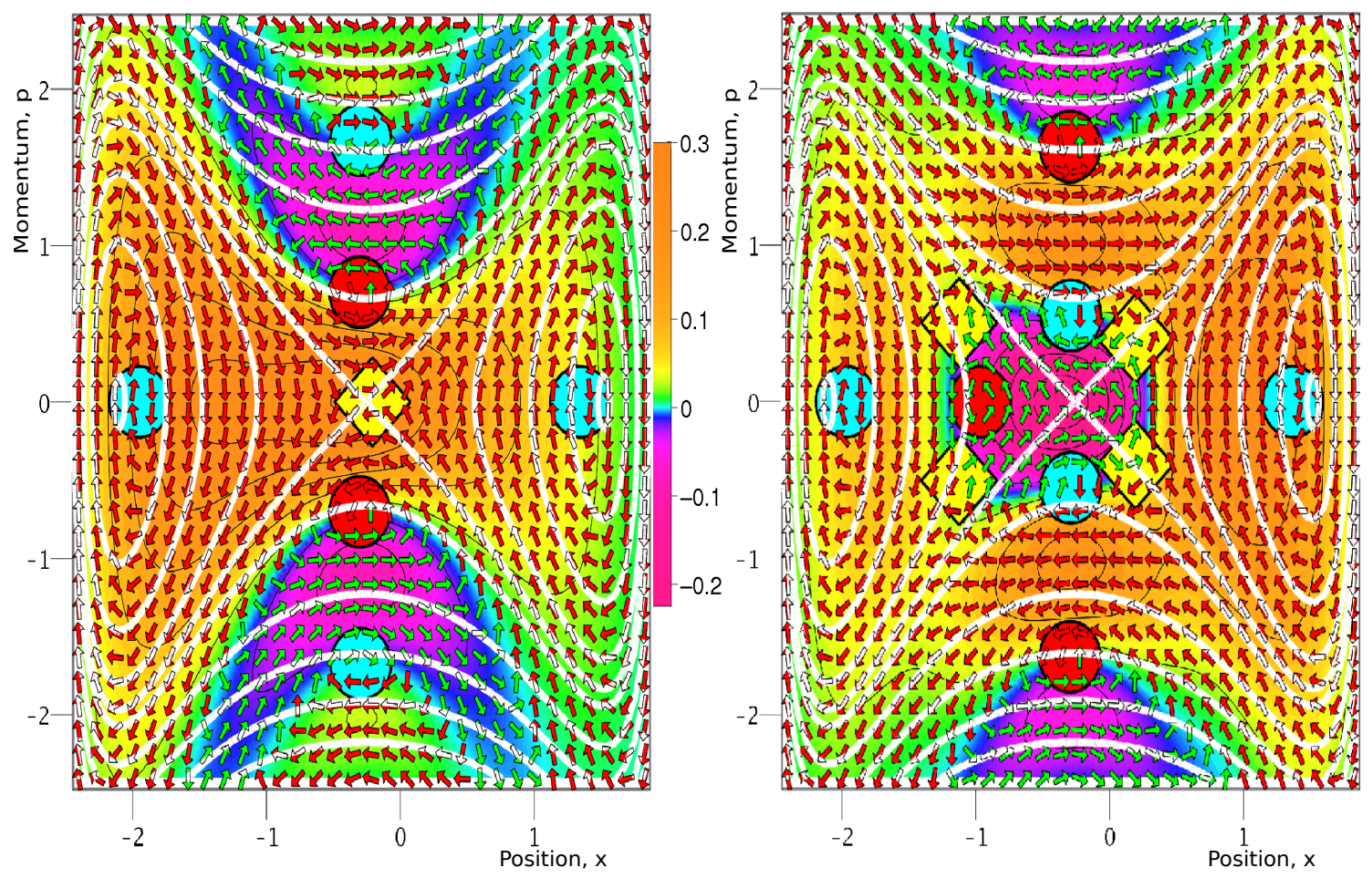

FIG. S. The Wigner flow of the eigenstates $\psi_{0}$ and $\psi_{1}$ displays the symmetries of equations $(9)$ and $(10)$. The same parameters and color coding as in Fig. 3 are used. 